\title{
RELACIÓN DE LOS FACTORES CLINICOS, ECOGRÁFICOS E INMUNOSEROLÓGICOS PREOPERATORIOS CON LA HISTOLOGÍA Y EL ESTADÍO DE LAS NEOPLASIAS TESTICULARES.
}

\author{
Ramón Diz Rodríguez, M iguel Vírseda Chamorro, Pablo Q uijano Barroso, Carlos Alpuente \\ Román, Domingo Sáez Benito y Pedro Paños Lozano.
}

Servicio de Urología. Hospital C entral de la Defensa. Madrid. España

\begin{abstract}
Resumen.- O BJETIVO: Determinar y cuantificar la relación que existe entre los datos clínicos ecográficos e inmunoserológicos y el tipo histológico y estadío de las neoplasias testiculares.

MÉTO DO S: Se analizó una cohorte de 80 casos sometidos a orquiectomía por neoplasia testicular. La media de edad de los pacientes fue de 30,4 años. Se recogió de manera retrospectiva los antecedentes del pacientes, la naturaleza del primer síntoma, el tiempo desde el primer síntoma hasta la intervención, los da tos de la exploración clínica y ecografía y los niveles preoperatorios de alfa-fetoproteína y beta HCG.

RESULTADOS: Los pacientes con Tumor de No Seminma toso de Células G erminales (TN SC G) tenían una edad media $(23,7$ años) significativa mente menor que la de los pacientes con seminoma puro $(41,3$ años), y ésta, a su vez, significativamente menor que la edad de los pacientes con Tumores No Germinales
\end{abstract}

M iguel Vírseda C hamorro

C/ Argentina 4, 10-1 a

28027 M adrid. (España)

e-mail: bgemeli@terra.es

Trabajo recibido: 3 de septiembre 2004
(TN G) (50,7 años). La presencia como síntoma inicial de malestar general o dolor lumbar aumentó el riesgo relativo de padecer un tumor en un estadío avanzado (superior al estadío I) 2,56 veces. La existencia de ginecomástia aumentó 16,5 veces el riesgo relativo de padecer un $T N G$, debido a la presencia en este grupo de los tumores de Leydig. La observación de nódulos heterogéneos en la ecografía incrementó el riesgo de padecer un TG NS 4,5 veces. La elevación preoperatoria de la alfa-fetoproteína descartó la existencia de un seminoma y de un $T N G$, mientras que la elevación preoperatoria de la beta HCG aumentó el riesgo de padecer un TGNS 3,21 veces. No se observó una relación significativa entre los niveles preoperatorios de a mbos marcadores y el estadío tumoral.

CON CLUSIO NES: La existencia de ginecomastia, la edad, la visualización de nódulos heterogéneos en la ecografía y los niveles preoperatorios de alfa-fetoproteina y beta HCG son datos relevantes relacionados con el tipo histológico de tumor. La sensación de dolor lumbar o malestar general es un dato clínico relacionado con el estadío tumoral.

Palabras clave: Tumor de testículo. G inecomastia. Histología. Esta dio tumoral. Ecografía. Alfa-fetoproteína. Beta $H C G$.

Resumen.- $O$ BJEC TIVES: To evalua te and quantify the association between clinical, ultrasound and immunoserological data and histologic type and stage of testicular tumors. 
METHODS: We analyze a cohort of 80 patients who underwent orchiectomy for testicular neoplasia. M ean patient age was 30.4 years. Past medical history, first symptoms, time from first symptom to operation, physical examination and ultrasound data, and preoperative serum levels of alpha-fetoprotein and beta HCG were retrospectively collected.

RESULTS: Patients with non seminomatous germ cell tumor (N SG CT) had a significantly lower mean age (23.7 $\mathrm{yr}$.) than patients with pure seminoma (41.3 yr.), and these latter a significantly lower age than patients with non germinal tumors (50.7 yr.). Initial presentation with general malaise or lumbar pain increased 2.56 times the relative risk of having a tumor in advanced stage (higher than stage I). Presence of gynecomastia increased 16.5 times the relative risk of having a non germ cell tumor, due to the inclusion of Leydig's tumors in this group. Detection of heterogeneous nodules by ultrasound increased 4.5 times the risk of having a non seminomatous germ cell tumor. Preoperative elevation of alpha-fetoprotein ruled out the existence of seminoma and non germ cell tumor; whereas preoperative elevation of beta HCG increased 3.21 times the risk of having a non seminomatous germ cell tumor. No significant association was shown between preoperative tumor markers and tumor stage.

CON C LUSIO N S: The existence of gynecomastia, age, detection of heterogeneous nodules on ultrasound, and preoperative alpha-fetoprotein and beta HCG are relevant data in relation to histological type of tumor. Lumbar pain or malaise a re clinical data associated with tumor stage.

Keyw ords: Testicular tumor. G ynecomastia. $H$ istology. Tumor stage. Ultrasound. Alpha-feto protein. Beta HCG.

\section{INTRODUCCIÓN}

Los tumores de testículo representan alrededor de $1,5 \%$ de las neoplasias del varón (1), y parece que su incidencia está aumentando particularmente en los paises occidentales (2). En general, estos tumores muestran una excelente tasa de curación. Entre los factores que influyen en su pronóstico se encuentran el tipo histológico de tumor y el estadío en el que son diagnosticados. Se considera que los seminomas son de mejor pronóstico que los tumores no seminomatosos (3), y que cuando el tumor está confinado al testículo (estadio I), los pacientes pueden ser tratados satis- factoriamente en un centro no especializado en este tipo de neoplasias, mientras que en estadíos más avanzados su tasa de supervivencia depende del número de pacientes tratados en la institución (4).

Los primeros datos de los que se dispone cuando se sospecha un tumor testicular son la historia clínica, la ecografía y los niveles plasmáticos previos a la cirugía de alfa-fetoproteina y beta HCG. El tipo de tumor sólo puede ser diagnosticado mediante el estudio anatomo-patológico, y el estadío tumoral precisa para su tipificación, de la realización de exploraciones complementarias como el TAC abdominal, la radiografía simple y el TAC torácico (5). Los resultados definitivos suelen demorarse cierto tiempo, por lo que es interesante investigar si con los datos proporcionados por la clínica, la ecografía y los niveles previos de los marcadores tumorales, sería posible tener una primera impresión sobre el tipo histológico y el estadío tumoral, para de esta forma efectuar una primera valoración pronóstica.

El objetivo de este trabajo es determinar y cuantificar la posible relación entre los parámetros proporcionados por la historia clínica, le ecografía y la concentración sérica previa de los marcadores tumorales alfa-fetoproteina y beta-HCG con respecto al tipo histológico y estadío tumoral de las neoplasias testiculares.

\section{MATERIAL Y MÉTODOS}

Se realizó un estudio longitudinal retrospectivo en una cohorte de 80 casos intervenidos de orquiectomía en nuestro centro por padecer neoplasia testicular. Los datos se recogieron de los registros en un intervalo de tiempo de 20 años (desde enero de 1984, hasta enero de 2004).

La edad media de los pacientes fue de 30,4 años (desviación típica: 14,7 años). Los datos recogidos en el estudio fueron: los antecedentes de criptorquidia, el síntoma inicial, el tiempo transcurrido desde la aparición del primer síntoma hasta la intervención, la presencia de ginecomastia, y el testículo afectado. En la exploración clínica se valoró la palpación del nódulo tumoral. El estudio ecográfico se efectuó mediante ecógrafos de alta resolución y frecuencia de entre 7 y 10 MegaHerztios. En este estudio se comprobó la presencia de nódulos, su ecogenicidad, la existencia de calcificaciones y de lesiones asociadas como hidrocele o signos compatibles con orquiepididi- 
mitis. Las concentraciones séricas de de alfa-fetoproteina y beta HCG antes de la orquiectomía se a nalizaron mediante electroquimioinmunoanálisis. Se consideraron normales valores de alfa fetoproteina inferiores a $13 \mathrm{ng} / \mathrm{ml}$ y de beta HCG inferiores a $5 \mathrm{mUl} / \mathrm{ml}$. En el estudio anatomo-patológico se determinó el tipo de tumor según la clasificación de Mostofi $(6,7)$. Para el diagnóstico de extensión se realizó una radiografía simple de torax, y un TAC torácico y abdominal. El estadío tumoral se determinó de acuerdo con la clasificación del Royal Madsen Hospital (8).

Los datos de los pacientes fueron a Imacenados en una base de datos ACCESS y posteriormente importados al programa informático SPSS (versión 10), para su procesamiento estadístico. En el análisis estadístico se utilizó el test de la chi-cuadrado de Pearson para comparar dos variables cualitativas, el test de comparación de medias de la t de Student en el caso de dos variables cuantitativas, y el análisis de la variancia (ANOVA) con el test de contraste post hoc de Sheffe para comparar más de dos variables cuantitativas. El nivel de significación se fijo en el 95\% bilateral.

\section{RESULTADOS}

\section{Estadística descriptiva}

La sintomatología inicial se muestra en la Tabla I. El síntoma inicial más común fue la presencia

TABLA I. SÍN TO MAS IN IC IALES DE LOS TUMO RES TESTIC ULARES

\begin{tabular}{|lr|}
\hline \multicolumn{1}{|c|}{ Síntoma } & Porcentaje \\
\hline Tumoración testicular & $54 \%$ \\
Dolor en testículo & $14 \%$ \\
Tumoración y dolor testicular & $5 \%$ \\
A umento de consistencia & $2 \%$ \\
Tumoración y aumento de consistencia & $4 \%$ \\
Inflamación testicular & $7 \%$ \\
Traumatismo testicular & $2 \%$ \\
Hidrocele o quiste de epidídimo & $4 \%$ \\
Ginecomastia & $2 \%$ \\
Dolor lumbar & $2 \%$ \\
Malestar general & $2 \%$ \\
Dolor lumbar y malestar general & $2 \%$ \\
Total & $100 \%$ \\
\hline
\end{tabular}

de una tumoración testicular ( $54 \%$ de los casos), seguido del dolor testicular aislado (14\% de los casos). El tiempo transcurrido desde la aparición del primer síntoma hasta la orquiectomía fue de 340,8 días (desviación típica 764,6 días), aunque la mediana (tiempo transcurrido para la mitad de los pacientes), fue de 56 días. El resto de los datos clínicos se muestran en la Tabla II. El el 93\% de los casos se palpó un nódulo en el testículo, mientras que los antecedentes de criptorquidia o la presencia de ginecomastia fue baja.

Los datos ecográficos se muestran en la Tabla III. El hallazgo más frecuente fue la observación de un nódulo único hipoecogénico. Los niveles séricos de los marcadores tumorales antes de la intervención se muestran en la Tabla IV. En el $48 \%$ de los casos los niveles de alfa-fetoproteína estaban elevados. El porcentaje de pacientes con elevación de la beta HCG preoperatoria fue del $56 \%$. Un $69 \%$ de los pacientes tenían al menos uno de los marcadores elevado (estadio $S_{1}$ ). En ningún caso los niveles de alfa-fetoproteína fueron superiores o iguales a $5.000 \mathrm{mUl} / \mathrm{ml}$ y los de beta HCG superiores 0 iguales a $1.000 \mathrm{mUl} / \mathrm{ml}$. Es decir, en ningún caso el estadío fue superior a $S_{1}(8)$.

La estirpe tumoral se muestra en la Tabla V. Las neoplasias más comunes fueron los tumores germinales puros no seminomatosos (33\%), seguidos del seminoma puro (29\%). El estadío tumoral se muestra en la Tabla VI. El estadío más común fue el estadio I (62\% de los casos).

\section{Estadística inferencial}

Relación con la estirpe tumoral

Se comprobó una relación significativa entre la edad y la estirpe tumoral. Los tumores de células germinales formados por elementos no seminomatosos

TABLA II. DATO S DE LA HISTO RIA C LÍN IC A

\begin{tabular}{|lr|}
\hline \multicolumn{1}{|c|}{ Hallazgos } & Porcentaje \\
\hline A ntecedente de criptorquidia & $7 \%$ \\
Ginecomastia & $4 \%$ \\
Palpación de nódulo en testículo & $93 \%$ \\
Testículo afectado & \\
Derecho & $56 \%$ \\
Izquierdo & $44 \%$ \\
\hline
\end{tabular}


(puros o mixtos) se presentaron en pacientes significativamente más jóvenes que las otras dos estirpes tumorales, siendo los pacientes de mayor edad los que padecían tumores no germinales (Figura 1). La diferencia del tiempo hasta el diagnóstico entre los distintos tipos de tumores no fue significativa. Tampoco hubo diferencias significativas entre la estirpe tumoral y el síntoma inicial referido por los pacientes. El único dato clínico significativo fue la presencia de ginecomastia que ocurrió en el 33\% de los tumores no germinales, frente a solamente el $2 \%$ de los tumores germinales. El riesgo relativo de padecer un tumor no germinal si existe ginecomastia es de 16,5 veces mayor.

En relación a los datos ecográficos, únicamente se comprobó una diferencia significativa respecto a la presencia de nódulos heterogéneos (presentes en el $36 \%$ de los tumores no seminomatosos, frente a sólo el $8 \%$ de los seminomas puros). La presencia de un nódulo heterogéneo aumenta el riesgo relativo de tener un tumor no seminomatoso 4,5 veces.

La relación entre el nivel de los marcadores tumorales y la estirpe histológica de tumor se muestran en la Tabla VII. Las diferencias en el porcentaje de marcadores normales fueron significativamente diferentes según el tipo de tumor. Ningún paciente con tumor no germinal presentó marcadores elevados. Tampoco se elevaron los niveles de alfa-fetoproteina en los pacientes con seminoma puro. El $74 \%$ de los pacientes con tumores germinales no seminomatosos tenían elevada la beta HCG frente al $23 \%$ del resto de tumores. Es decir, los pacientes con una una beta HCG elevada tienen 3,21 veces más posibilidades de que el tumor sea del tipo germinal no seminomatoso.

TABLA III. DATOS ECO G RÁFIC OS

\begin{tabular}{|lr|}
\hline \multicolumn{1}{|c|}{ Hallazgos } & Porcentaje \\
\hline N ódulo único hipoecogénico & $54 \%$ \\
N ódulos múltiples hipoecogénicos & $14 \%$ \\
N ódulo único hiperecogénico & $5 \%$ \\
N ódulo único heterogéneo & $2 \%$ \\
N ódulos múltiples heterogéneos & $4 \%$ \\
N ódulo heterogéneo con calcificaciones & $7 \%$ \\
Hidrocele y nódulo hipoecogénico & $2 \%$ \\
O rquiepididimitis & $4 \%$ \\
Total & $100 \%$ \\
\hline
\end{tabular}

Relación con el estadío tumoral

No se observó ninguna diferencia significativa entre el estadío tumoral y la edad de los pacientes. La diferencia respecto del tiempo diagnóstico tampoco fue significativa. Respecto al síntoma inicial, se observó que el $100 \%$ de los pacientes con malestar general o dolor lumbar presentaban un estadío tumoral avanzado, frente a un $39 \%$ con estadío tumoral avanzado en ausencia de estos síntomas (diferencias significativas). El riesgo relativo de estadío tumoral avanzado con la presencia de estos síntomas fue de 2,56 veces más.

En relación a los datos ecográficos, no se observaron diferencias significativas respecto al estadio tumoral. El nivel de marcadores tumorales tampoco mostró diferencias significativas.

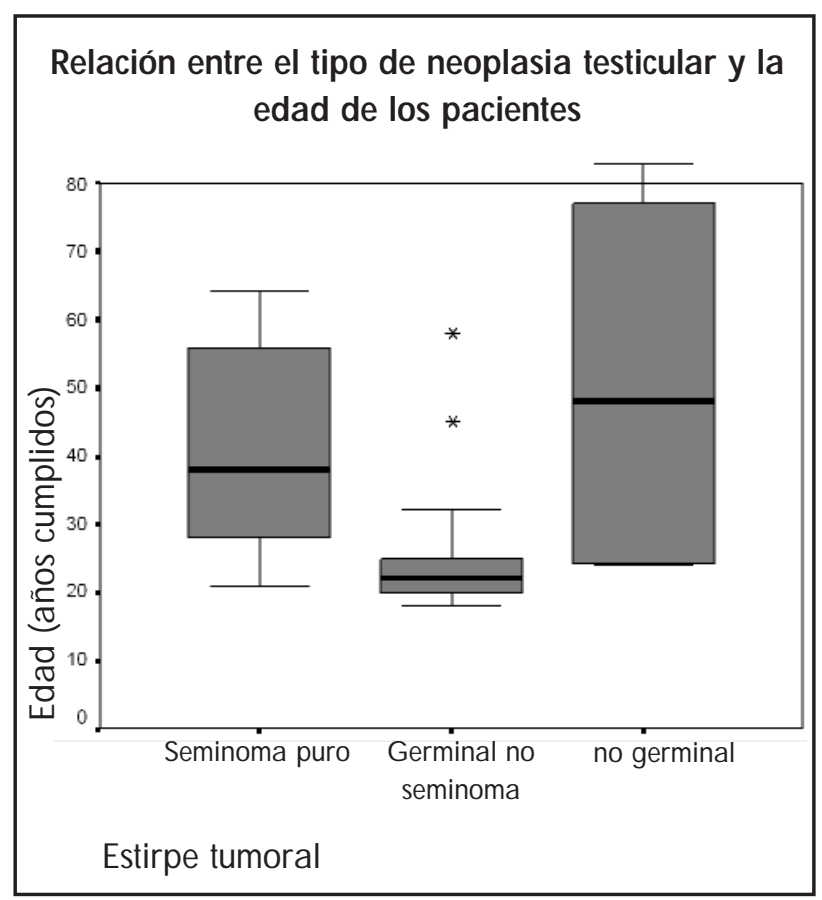

FIG URA 1. Diagrama de caja que muestra la distribución de edades de los tres tipos histológicos de tumor testicular. La linea central representa a la mediana de cada grupo, y la longitud de la caja es proporcional a su amplitud intercuartil. La media de los pacientes con neoplasia germinal no seminomatosa fue de 23,7 Años (Desviación típica: 7,0), la de los pacientes con seminoma de 41,3 años (desvación típica: 14,3) y la de los pacientes con tumor germinal no seminomatoso de 50,7 años (desviación típica 30,7 años). Las diferencias entres los tres grupos fueron significativas (AN O VA $y$ test post hoc de Sheffe). 
TABLA IV. VALO RES SÉRIC OS DE LOS MARCADO RES TUM O RALES AN TES DE LA O RQ UIEC TO M ÍA

\begin{tabular}{|lcr|}
\hline Marcador & Media y (desviación típica) & Porcentaje de niveles elevados \\
\hline Alfa feto-proteína & $141,29(340,66) \mathrm{ng} / \mathrm{ml}$ & $48 \%$ \\
Beta HCG & $158,04(507,43) \mathrm{mUl} / \mathrm{ml}$ & $56 \%$ \\
\hline
\end{tabular}

\section{DISCUSIÓN}

En nuestro estudio la edad media de aparición de los tumores germinales no seminomatosos (23,7 años), fue significativamente menor que la de los seminomas puros (41,3 años) y la de éstos menor que la de los no germinales $(50,7$ años). Estos datos coinciden por los publicados en otras series. A si Post et al,
(10) en una revisión de tumores testiculares encuentran que en varones menores de 30 años son más frecuentes los tumores no seminomatosos, entre los 30 y 65 años los seminomas, y a partir de los 65 años los linfomas (que serían los tumores no germinales más frecuentes). En España, Armora et al (11) indican que la edad media de los pacientes con seminoma fue de 34 años, frente a los 26 años de los pacientes con tumo-

TABLA V. ESTIRPE HISTO LÓ GICA DEL TUMOR

\begin{tabular}{|c|c|c|c|}
\hline Estirpe tumoral & Frecuencia absoluta & & Porcentaje \\
\hline \multicolumn{4}{|l|}{ Seminomas puros } \\
\hline Seminoma clásico & 19 & $24 \%$ & \\
\hline Seminoma anaplásico & 3 & $4 \%$ & \\
\hline Seminoma espermatocítico & 1 & $1 \%$ & \\
\hline Total seminomas & 23 & & $29 \%$ \\
\hline \multicolumn{4}{|l|}{ O tros tumores germinales puros } \\
\hline Carcinoma embrionario & 22 & $27 \%$ & \\
\hline Coriocarcinoma & 1 & $1 \%$ & \\
\hline Teratoma maduro & 3 & $4 \%$ & \\
\hline Teratoma inmaduro & 1 & $1 \%$ & \\
\hline $\begin{array}{l}\text { Total otros tumores germinales puros } \\
\text { Seminoma mixto }\end{array}$ & 27 & & $33 \%$ \\
\hline Seminoma + Ca embrionario & 3 & $4 \%$ & \\
\hline Seminoma + teratoma & 2 & $2 \%$ & \\
\hline Seminoma + teratocarcinoma & 8 & $10 \%$ & \\
\hline $\begin{array}{l}\text { Total seminomas mixtos } \\
\text { O tros tumores germinales mixtos }\end{array}$ & 13 & & $16 \%$ \\
\hline Teratocarcinoma & 12 & $15 \%$ & \\
\hline Ca embrionario + coriocarcinoma & 1 & $1 \%$ & \\
\hline $\begin{array}{l}\text { Total otros tumores germinales mixtos } \\
\text { Tumores no germinales }\end{array}$ & & & $16 \%$ \\
\hline Tumor de Leydig & 2 & $3 \%$ & \\
\hline Linfoma de alto grado & 2 & $3 \%$ & \\
\hline Total tumores no germinales & 5 & & $6 \%$ \\
\hline Total & 80 & & $100 \%$ \\
\hline
\end{tabular}


res germinales no seminomatosos.

La criptorquidia sería el único factor de riesgo claramente relacionado con el cancer testicular (1). El antecedente de criptorquidia estuvo presente en el $7 \%$ de la muestra. En otras series esta dato aparece en el $11 \%$ de los casos (12). Para algunos autores, el riesgo de seminoma asociado a criptorquidia, sería mas alto que para otros tipos de tumor (13). En nuestro estudio no se apreció ninguna relación entre el antecedente de criptorquidia y el tipo de tumor. Raina et al (14) en una serie de 24 tumores en testículos criptorquidicos encuentran la misma proporción de seminomas que de tumores germinales no seminomatosos.

El retraso medio desde el primer síntoma hasta la orquiectomía, fue en nuestra serie de 340,8 días, aunque la mitad de los pacientes fueron intervenidos en menos de 56 días. En otras series el tiempo medio transcurrido hasta el diagnóstico se estima en 98 días al que hay que sumar 37 días hasta la orquiectomía (15). Estos tiempos se han ido reduciendo con los años. En nuestra serie no encontramos relación entre el periodo de tiempo transcurrido hasta la intervención y el estadío tumoral. Hernes et al (16) si encuentran relación, pero únicamente en los seminomas. Según estos autores un retraso de más de 112 días se asocia a una mayor probabilidad de un estadío avanzado. Es posible que la distribución asimétrica respecto al tiempo de espera en nuestra serie haya enmascarado esta relación.

El dato clínico más frecuente en nuestra serie fue la existencia de una tumoración testicular indolora, referida por el $54 \%$ de los pacientes y palpable por el explorador en el $93 \%$ de los casos. El dolor testicular como signo inicial (aislado o asociado a tumoración testicular) solo fue referido por el $19 \%$ de los pacientes. Estos datos coinciden con los observados en otras series (16).

TABLA VI. ESTADÍO TUMO RAL

\begin{tabular}{|lr|}
\hline \multicolumn{1}{|c|}{ Estadío tumoral } & Porcentaje \\
\hline Estadio I & $62 \%$ \\
Estadio II & $29 \%$ \\
Estadio III & $7 \%$ \\
Estadio IV & $2 \%$ \\
Total & $100 \%$ \\
\hline
\end{tabular}

La ginecomastia es un signo poco frecuente. Se estima que ocurre en el $7 \%$ de los pacientes con tumores testiculares (17). En nuestra serie apareció en el $33 \%$ de los tumores no germinales, (correspondiendo a tumores de Leydig), frente a sólo el $2 \%$ de los germinales. Su presencia aumentó la probabilidad de que se tratara de un tumor de estirpe no germinal 16,5 veces. La ginecomastia es típica de los tumores de células de Leydig, y se supone que está relacionada con la elevación de los niveles de estradiol plasmático (18). Sin embargo, es posible que otros tumores, como los secretores de HCG, entre los que se encuentra el coriocarcinoma, también la originen. En estos casos, la aparición de ginecomastia es un factor de mal pronóstico (19). A mbas condiciones estuvieron presentes en otro paciente de nuestra serie con ginecomastia.

El dolor lumbar estuvo presente, como síntoma inicial, en el $4 \%$ de nuestra serie. 0 tros autores elevan su incidencia hasta el $11 \%$ de los casos (17). Este síntoma y la sensación de malestar general constituyeron un factor de riesgo de padecer un estadio tumoral avanzado. El riesgo de neoplasia extratesticular aumentó 2,56 veces. Cantwell et al, (20), tambien encuentran nódulos paraaóticos en sus nueve casos de pacientes con dolor lumbar y neoplasia testicular. Estos a utores recomiendan explorar los testículos a todos los pacientes jóvenes que refieran dolor lumbar.

Se considera que la ecografía testicular tiene una sensibilidad de casi el $100 \%$ para el diagnóstico de los tumores testiculares $(21,22)$. Algunos autores indican que los seminomas son generalmente lesiones homogéneas y bien definidas, mientras que los tumores testiculares no seminomatosos puros 0 mixtos tienen una apariencia mucho mas variada (23). 0 tros autores, por el contrario, consideran que sólo hay una débil tendencia en los seminomas a producir imágenes regulares, frente a la irregularidad del resto de los tumores seminomatosos, y que estas diferencias carecen de utilidad para distinguir entre los distintos tipos de tumores (24). En nuestro estudio descubrimos una asociación significativa entre la presencia de nódulos de características ecográficas heterogéneas y los tumores no seminomatosos, de manera que si se observan imágenes de este tipo, aumenta la probabilidad de que se trate de un tumor no seminomatoso 4,5 veces. Gallardo et al (22) tambien refieren que las imágenes de aspecto quístico o hiperecoico aparecen en un $30 \%$ de su serie de tumores no seminomatosos, frente a ningún caso de los seminomatosos. 
En nuestra serie se comprobó una elevación de los niveles preoperatorios de alfa-fetroproteína en el $48 \%$ de los casos y de beta HCG en el $56 \%$, estando elevado al menos un marcador en el $69 \%$ de los pacientes. Está universalmente aceptado que existe una relación entre los niveles plasmáticos de alfa-fetoproteina y beta HCG y la estirpe tumoral. A sí los seminomas puros no producen alfa-fetoproteina en ningún caso, mientras que el procentaje de este tipo de tumor secretor de beta HCG puede elevarse en el curso de la enfermedad hasta el $30 \%(25)$, (26). En nuestra serie el porcentaje de seminomas con niveles de beta HCG elevados fue del $27 \%$.

La alfa fetoproteína es producida por células endodérmicas embrionarias, por lo que únicamente está elevada en tumores con células del seno endodérmico (como el tumor de yolk sac). En el resto de los tumores germinales no seminomatosos su incremento dependerá de la existencia de este tipo de células (27), (28). En nuestros casos, este marcador estaba elevado preoperatoriamente en el $87 \%$ de los tumores germinales mixtos no seminomatosos, en el $71 \%$ de los germinales puros no seminomatosos, y en el el $67 \%$ de los seminomas mixtos. 0 tros autores refieren un porcentaje de aumento de este marcador en los tumores germinales no seminomatosos, de entre un 33\% (29), y un $70 \%(30)$, siendo más frecuente encontrar niveles anormales en el carcinoma embrionario y en el teratoma (31).

La producción de beta HCG tiene lugar en las células multinucleadas del sincitiotrofoblasto, por 10 que este marcador está elevado en el $100 \%$ de los coriocarcinomas $(27,28)$. En el resto de los tumores no seminomatosos sus niveles dependerán de la presencia de este tipo de células. En nuestro estudio este marcador se elevó en el $86 \%$ de los tumores germinales puros no seminomatosos, el $78 \%$ de los germinales mixtos no seminomatosos y en el $56 \%$ de los seminomas mixtos. En conjunto, los pacientes con una beta HCG elevada tienen 3,21 veces más probabilidades de que el tumor sea germinal no seminomatoso que de otro tipo. 0 tros autores refieren un incremento de este marcador en los tumores germinales no seminomatosos, entre el $34 \%(29)$, y el $60 \%$ (30) de los casos.

La elevación de alguno de los dos marcadores tumorales tuvo lugar en el $100 \%$ de los tumores mixtos no seminomatosos, el $93 \%$ de los tumores germinales puros no seminomatosos y el $89 \%$ de los seminomas mixtos, mientras que sólo tuvo lugar en un $27 \%$ de los seminomas puros (a expensas del aumento de la beta HCG ) y en ningún caso de los tumores no germinales. 0 tros autores, refieren un incremento en los niveles de algún marcador en tumores germinales no seminomatosos de entre el $79 \%$ (32), y el $90 \%$ (30) de los casos.

Es decir, la determinación de los marcadores tumorales es útil para el diagnóstico de neoplasia tumoral, principalmente para los tumores germinales que no son seminomas puros. Aunque no se debe olvidar que existe un cierto porcentaje (un $7 \%$ de tumores germinales puros no seminomatosos y un $11 \%$ de seminomas mixtos, en nuestra serie), cuyos marcadores también fueron negativos. Por otra parte, esta determinación no es útil para el diagnóstico de los seminomas puros ni para los tumores testiculares no germinales.

TABLA VII. RELACIÓ N EN TRE LO S VALO RES PREO PERATO RIO S DE LOS MARCADO RES TUM O RALES Y LA ESTIRPE HISTO LO GICA TUM O RAL

\begin{tabular}{|lrrr|}
\hline \multicolumn{1}{|c|}{ Estirpe tumoral } & $\begin{array}{c}\text { Porcentaje de pacientes } \\
\text { con alfa-fetoproteina } \\
\text { elevada* }\end{array}$ & $\begin{array}{c}\text { Porcentaje de } \\
\text { pacientes con beta } \\
\text { HCG elevada* }\end{array}$ & $\begin{array}{r}\text { Porcentaje de } \\
\text { pacientes con algún } \\
\text { marcador elevado* }\end{array}$ \\
\hline Seminoma puro & $0 \%$ & $27 \%$ & $27 \%$ \\
O tros tumores germinales puros & $71 \%$ & $85 \%$ & $93 \%$ \\
Seminoma mixto & $67 \%$ & $56 \%$ & $89 \%$ \\
O tros tumores germinales mixtos & $87 \%$ & $78 \%$ & $100 \%$ \\
Tumores no germinales & $0 \%$ & $0 \%$ & $0 \%$ \\
\hline
\end{tabular}


Por último, hay que señalar que en nuestro estudio no encontramos relación entre el nivel preoperatorio de ambos marcadores y el estadío tumoral. G erl et al (33), tampoco encuentran relación entre estos niveles y la evolución de la enfermedad. Sin embargo tanto Huertas et al (34), como Fernández et al (29), refieren hallar una relación significativa entre los niveles de estos marcadores y el estadío tumoral. Es posible que está relación sea más evidente cuando los niveles de ambos marcadores soprepasan el estadío S.

\section{BIBUOGRAFIA y LECTURAS RECOMENDADAS (*lectura de interés y **lectura fundamental)}

1. SCHOTTENFIELD, D.; WARSHAUWE, M.E.; SHERLOCJ, S. y cols.: "The epidemiology of testicular cancer in young adults". Am. J. Epidemiol., 112: 232, 1980.

2. HUYGHE, E.; MATSUDA, T.; THONNEAU, P.: "Increasing incidence of testicular cancer worldwide. A review". J. Urol., 170: $5,2003$.

*3. International Germ Cell Cancer Collaborative Group. "International Germ Cell Consensus Classification: a prognostic factor-based staging system for metastasis germ cell cancers". J. Clin. Oncol., 15: 594. 1997.

4. COLLETTE, L.; SYLVESTER, R.J.; STENNING, S.P. y cols.: "Impact of the treating institution on survival of patients with poor-prognosis non-seminoma". J. Natl. Cancer Inst., 91: 839, 1999.

5. ELLIS, J.H.; BLIES, J.R.; KOPECKY, K.K. y cols.: "Comparison of NMR and CT imaging in the evaluation of metastasic retoperitoneal lymphadenopathy from testicular carcinoma". J. Comp. Assist. Tomogr., 8: 709, 1984.

6. MOSTOFI, F.K.; SOBIN, L.H.: "International histological classification of tumor of the teste". WHO, Geneve, 1977.

7. MOSTOFI, F.K.; SESTERHENN, I.A.: "Histological typing of the testis tumors". WHO International Histological Classification Tumors 2nd Edition . Springer-Verlag: Berlin, 1998.

8. PECKHAM, M.J.; MCELWAIN, T.J.; BARRET, A y cols.: "Combined management of malignan teratoma of the testis". Lancet, 2: 267, 1979.

9. SOBIN, L.H.; WITTEKIND, Ch (eds).: "UICC: TNM Classification of malignant tumours". 6th edition, Wiley-Liss Inc: New York, 2002:

10. POST, P.N.; CASPARIE, M.K.; TEN KATE, F.J. y cols.: "The epidemiology of tumors of the testes in the Netherlands: accurate rendering by the Registry of Histopathology and Cytopathology". (PALGA) Ned Tijdschr Geneeskd., 148: 1150, 2004.

*11. ARMORA MANI, J.; RIERA CANALS, L.; FRANCO MIRANDA, E. y cols.: "Tumores germinales de testículo. Aportación de nuestra casuística y revisión de la literatura" Actas Urol. Esp., 14: 319, 1990.

*12. GERMA, J.R.; GARCIA DEL MURO, X.; MAROTO, P. y cols.: "Grupo Germinal. Patrones clínicos y resultados terapéuticos obtenidos en el cancer testicular de células germinales en España basados en una serie consecutiva de 1.250 pacientes". Med. Clin. (Barc)., 116: $481,2001$.

13. COUPLAND, C.A,; CHILVERS, C.E.; DAVEY, G. y cols.: "Risk factors for testicular germ cell tumours by histological tumour type". United Kingdom Testicular Cancer Study Group. Br J Cancer., 80: 1859, 1999.
14. RAINA, V.; SHULKLA, N.K.; GUPTA, N.P., y cols.: "Germ cell tumours in uncorrected cryptorchid testis at Institute Rotary Cancer Hospital, New Delhi”. Br. J. Cancer., 71: 380, 1995.

15. HERNES, E.H.; HARSTAD, K.; FOSSA, S.D.: "Changing incidence and delay of testicular cancer in southern Norway (1981-1992)". Eur. Urol., 30: 349, 1996.

**16. RICHIE, J.P.: "Neoplasm of the testis". In: Campbell's Urology 7th ed. Walsh PC et al, (edts): p.2411-2452, Wb Saunder Co: Philadelphia, 1997.

17. WANDERAS, E.H.; TRETLI, S.; FOSSA, S.D.: "Trends in incidence of testicular cancer in Norway". Eur. J. Cancer, 31: 2044, 1995.

18. CATALA BAUSET, M.; GIRBES BORRAS, J.; CARMENA-RAMON, R. y cols. "Ginecomastia y tumor de Leydig". An. Med. Interna, 14: 131, 1997.

19. STEPANAS, A.V.; SAMAAN, N.A.; SCHULTZ, P.N y cols.: "Endocrine studies in testicular tumor patients with and without gynecomastia: a report of 45 cases". Cancer, 1: 369, 1978.

*20. CANTWELL, B.M.; MANNIX, K.A.; HARRIS, A.L.: "Back pain, a presentation of metastatic testicular germ cell tumours". Lancet., 31: 262, 1987.

21. DOHERTY, F.J.: "Ultrasound in the nonacute scrotum". In: Seminars in ultrasound CT and MRI. Raymond HW et al (eds). p.131-156.WB Saunders Co: New York, 1991.

22. GALLARDO AGROMAYOR, E.; PENA GOMEZ, E. y cols.: "Tumores testiculares. Hallazgos ecográficos". Arch .Esp. Urol., 49: 622, 1996.

23. WOODWARD, P.J.; SOHAEY, R.; O'DONOGHUE, M.J. y cols.: "From the archives of the AFIP: tumors and tumorlike lesions of the testis: radiologic-pathologic correlation". Radiographics, 22: 189, 2002.

24. KRAHE,T.; LEIPNER, N.; PORST, H.: "Echo morphology of testicular tumors". ROFO Fortschr Geb Rontgenstr Nuklearmed., 148: 609, 1988.

25. PEYRET, C.: "Tumeurs de testicle. Synthèse et recomendations en onco-urologie". Prog .Url., 2: 60, 1993.

26. RUTHER, U.; ROTHE, B.; GRUNERT, K. y cols.: "Role of human chorionic gonadotropin in patients with pure seminoma”. Eur. Urol., 26: 129, 1994.

*27. FOWLER, J.E. Jr.; SESTERHENN, I.; STUZMAN, R.E. y cols.: "Localization of alpha-fetoprotein and human chorionic gonadotropin to specific histologic types of nonseminomatous testicular cancer". Urology, 22: 649, 1983.

28. MORINAGA, S.; OJIMA, M.; SASANO, N.: "Human chorionic gonadotropin and alpha-fetoprotein in testicular germ cell tumors. An immunohistochemical study in comparison with tissue concentrations". Cancer, 52: 1281, 1983.

29. FERNANDEZ GOMEZ, J.M.; GUATE ORTIZ, J.L.; MARTÍN HUESCAR, A. y cols.: "Presentación clínica del cáncer germinal de testículo”. Arch. Esp. Urol., 55: 915, 2002.

30. JAVADPOR, N.: "The role of biologic markers in testicular cancer". Cancer, 45: 1755, 1980.

31. BELLET,D.; CAILLAUD, J.M.; DROZ, J.P, y cols.: "Marqueurs biologiques pour tumeurs du testicle. Leur use dans le diagnostique, pronostic et poursuite en 200 cases". Nouv Presse Med., 10: 3293, 1981.

**32. GERMA-LLUCH, J.R.; GARCIA DEL MURO, X.; MAROTO, P. y cols.: "Clinical pattern and therapeutical results achieved in 1490 patients with Germ-cell tumors of the testis: the experience of the Spanish Germ-cell Group (GG)". Eur. Urol., 42: 553, 2002.

33. GERL, A.; LAMERZ, R.; CLEMM,C. y cols.: "Does serum tumor marker half-life complement pretreatment risk stratification in metastatic nonseminomatous germ cell tumors?". Clin. Cancer Res., 2: 1565, 1996.

*34. HUERTAS MORA, R.A.; LARRODERA LOPEZ, L.; GÓMEZ MATOBELLA, I. y cols.: "Beta-HCG humana y alfa-fetoproteina como marcadores biológicos en tumores germinales testiculares". Rev. Esp. Oncol., 31: 321, 1984. 